\title{
Wilf-equivalence on $k$-ary words, compositions, and parking functions
}

\author{
Vít Jelínek* \\ Department of Applied Mathematics, Charles University, Prague \\ jelinek@kam.mff.cuni.cz \\ Toufik Mansour \\ Department of Mathematics, Haifa University, 31905 Haifa, Israel \\ toufik@math.haifa.ac.il \\ Submitted: May 9, 2008; Accepted: May 4, 2009; Published: May 11, 2009 \\ Mathematics Subject Classification: Primary 05A18; Secondary 05E10, 05A17, 05A19
}

\begin{abstract}
In this paper, we study pattern-avoidance in the set of words over the alphabet $[k]$. We say that a word $w \in[k]^{n}$ contains a pattern $\tau \in[\ell]^{m}$, if $w$ contains a subsequence order-isomorphic to $\tau$. This notion generalizes pattern-avoidance in permutations. We determine all the Wilf-equivalence classes of word patterns of length at most six.

We also consider analogous problems within the set of integer compositions and the set of parking functions, which may both be regarded as special types of words, and which contain all permutations. In both these restricted settings, we determine the equivalence classes of all patterns of length at most five.

As it turns out, the full classification of these short patterns can be obtained with only a few general bijective arguments, which are applicable to patterns of arbitrary size.
\end{abstract}

\section{Introduction}

In this paper, we study patterns avoidance in the domains of words, integer partitions, and parking functions. This can be seen as an extension of the frequently studied concept of pattern avoidance of permutation patterns. Our results extend previous work of Burstein [4], who described the equivalence classes of $k$-ary words of length at most 3 , and

\footnotetext{
${ }^{*}$ Supported by the project MSM0021620838 of the Czech Ministry of Education, and by the grant GD201/05/H014 of the Czech Science Foundation.
} 
of Savage and Wilf [14], who described the equivalence classes of integer compositions of length at most 3. Our classification is largely based on several new bijective arguments, inspired by the ideas from Krattenthaler [11], Backelin, West and Xin [3], and Jelínek and Mansour [9].

\section{$1.1 \quad k$-ary words}

Let $[k]=\{1,2, \ldots, k\}$ be a totally ordered alphabet of $k$ letters, and let $[k]^{n}$ denote the set of words of length $n$ over this alphabet.

Consider two words, $\sigma \in[k]^{n}$ and $\tau \in[\ell]^{m}$. Assume additionally that $\tau$ contains all letters 1 through $\ell$ (a word with this property will be called a pattern). We say that $\sigma$ contains an occurrence of $\tau$, or simply that $\sigma$ contains $\tau$, if $\sigma$ has a subsequence orderisomorphic to $\tau$, i.e., if there exist $1 \leq i_{1}<\ldots<i_{m} \leq n$ such that, for any two indices $1 \leq a, b \leq m, \sigma_{i_{a}}<\sigma_{i_{b}}$ if and only if $\tau_{a}<\tau_{b}$. If $\sigma$ contains no occurrences of $\tau$, we say that $\sigma$ avoids $\tau$. For a pattern $\tau$, let $[k]^{n}(\tau)$ denote the set of $k$-ary words of length $n$ which avoid the pattern $\tau$. Let $f_{\tau}(n, k)$ be the number of $\tau$-avoiding words in $[k]^{n}$, i.e., $f_{\tau}(n, k)=\left|[k]^{n}(\tau)\right|$.

We say that two patterns $\tau$ and $\tau^{\prime}$ are word-equivalent (or, more briefly, w-equivalent), and we write $\tau \stackrel{w}{\sim} \tau^{\prime}$, if for all values of $k$ and $n$, we have $f_{\tau}(n, k)=f_{\tau^{\prime}}(n, k)$.

There are two operations on words which trivially preserve the w-equivalence, called the reversal and the complement. The reversal of a word $\tau \in[k]^{m}$, denoted by $r(\tau)$, is obtained by writing the letters of $\tau$ in the reverse order, i.e., the $i$-th letter of $r(\tau)$ is equal to the $(m-i+1)$-th letter of $\tau$. The complement of a word $\tau$, denoted by $c(\tau)$, is obtained by turning $\tau$ "upside-down", i.e., a letter $j$ is replaced by the letter $\ell-j+1$, where $\ell$ is the largest letter of $\tau$. For example, if $\ell=3, m=4$, then $r(1232)=2321, c(1232)=3212$, $r(c(1232))=c(r(1232))=2123$. Clearly, $c \circ r=r \circ c$ and $r^{2}=c^{2}=(c \circ r)^{2}=i d$, so $\langle r, c\rangle$ is a group of symmetries of a rectangle.

Several authors have previously considered pattern avoidance in words $[1,2,4,5,10$, 13]. In 1998, Burstein [4] proved that $123 \stackrel{w}{\sim} 132$. In 2002, Burstein and Mansour [5] proved that $121 \stackrel{w}{\sim} 112$. By these two results we obtain that there are 3 w-equivalence classes of patterns of length three:

- 111,

- $112 \stackrel{w}{\sim} 121 \stackrel{w}{\sim} 122 \stackrel{w}{\sim} 211 \stackrel{w}{\sim} 212 \stackrel{w}{\sim} 221$

- $123 \stackrel{w}{\sim} 132 \stackrel{w}{\sim} 213 \stackrel{w}{\sim} 231 \stackrel{w}{\sim} 312 \stackrel{w}{\sim} 321$

\subsection{Compositions}

A composition $\sigma=\sigma_{1} \sigma_{2} \ldots \sigma_{m}$ of $n \in \mathbb{N}$ is an ordered collection of one or more positive integers whose sum is $n$. The numbers $\sigma_{1}, \ldots, \sigma_{m}$ are called parts of the composition. We let $C_{n}$ denote set of all compositions of $n$.

We say that the composition $\sigma \in C_{n}$ contains a pattern $\tau \in[\ell]^{s}$, if $\sigma$ contains a subsequence order-isomorphic to $\tau$. Let $C_{n}(\tau)$ denote the set of all the compositions in 
$C_{n}$ that avoid $\tau$. We say that two patterns $\tau$ and $\tau^{\prime}$ are composition-equivalent (or just c-equivalent), and we write $\tau \stackrel{c}{\sim} \tau^{\prime}$, if for all values of $n$, we have $\left|C_{n}(\tau)\right|=\left|C_{n}\left(\tau^{\prime}\right)\right|$. It is easy to see that every pattern is c-equivalent to its reversal. However, a pattern does not need to be c-equivalent to its complement.

Savage and Wilf [14] considered pattern avoidance in compositions for a single pattern $\tau \in S_{3}$ ( $S_{3}$ is the set of the permutations on three letters), and showed that the number of compositions of $n$ avoiding $\tau \in S_{3}$ is independent of $\tau$, that is, $123 \stackrel{c}{\sim} 213 \stackrel{c}{\sim} 132$. Recently, Heubach, Mansour and Munagi [7] showed that $112 \stackrel{c}{\sim} 121$ and $122 \stackrel{c}{\sim} 212$. These two results complete the classification of patterns of length three in compositions, and show they form exactly 4 c-equivalence classes:

- $123 \stackrel{c}{\sim} 213 \stackrel{c}{\sim} 132$,

- $112 \stackrel{c}{\sim} 121$,

- $122 \stackrel{c}{\sim} 212$,

- 111.

\subsection{Parking functions}

A word $\sigma \in[k]^{n}$ is called a parking function if for every $i=1, \ldots, n, \sigma$ has at least $i$ letters smaller or equal to $i$. Let $P F_{n}$ denote the set of parking functions of length $n$, and let $P F_{n}(\tau)$ be the set of the parking functions of length $n$ that avoid a pattern $\tau$. We say that two patterns $\tau, \tau^{\prime}$ are $p$-equivalent, denoted by $\tau \stackrel{p}{\sim} \tau^{\prime}$, if for every $n$, the two sets $P F_{n}(\tau)$ and $P F_{n}\left(\tau^{\prime}\right)$ have the same cardinality. Clearly, each pattern is p-equivalent to its reversal.

Although some enumerative aspects of parking functions have been previously studied $[6,12,15]$, we are not aware of any previous results dealing with pattern avoidance in this setting.

\subsection{Strong equivalence of words}

We now introduce an equivalence relation on words, which refines all the equivalences mentioned above. For a word $\sigma$ of length $n$, the content of $\sigma$ is the unordered multiset of the $n$ letters appearing in $\sigma$. In particular, two words have the same content, if one can be obtained from the other by a suitable rearrangement of letters.

We say that two patterns $\tau, \tau^{\prime}$ are strongly equivalent, denoted by $\tau \stackrel{s}{\sim} \tau^{\prime}$, if for every $k, n$ there is a bijection $f$ between $[k]^{n}(\tau)$ and $[k]^{n}\left(\tau^{\prime}\right)$ with the property that for every $\sigma \in[k]^{n}(\tau)$, the word $f(\sigma)$ has the same content as $\sigma$. Clearly, if two patterns are strongly equivalent, then they are also w-equivalent, c-equivalent and p-equivalent. Each pattern is strongly equivalent to its reversal, and if two patterns $\tau$ and $\tau^{\prime}$ are strongly equivalent, then their complements $c(\tau)$ and $c\left(\tau^{\prime}\right)$ are strongly equivalent as well.

In this note, we adapt previous results on fillings of diagrams [11], as well as results on pattern-avoidance in set partitions [9] to describe several types of content-preserving 
bijections between pattern-avoiding families of words. Using systematic computer enumeration of small patterns, we verify that these bijections, together with the reversal and complement operations, are sufficient to describe all the w-equivalent patterns of length at most six. Similarly, we verify that our results on strong equivalence describe all the c-equivalence and p-equivalence classes of patterns of size at most five. In particular, for patterns of size at most five, c-equivalence classes coincide with p-equivalence classes (but not with w-equivalence classes, because w-equivalence is closed under complementation and reversal, whereas p- and c-equivalence is only closed under reversal).

In the appendix, we briefly summarize the equivalence classes of small patterns, with respect to w-, c-, and p-equivalence. The full enumeration data and the source codes of the computer programs we used are available on the website of the second author $[16,17,18,19,20,21]$.

\section{Strongly equivalent families}

In this section, we use several techniques previously applied in the context of fillings of Ferrers diagrams to obtain classes of strongly equivalent words.

We may represent $k$-ary words of length $n$ as $0-1$ matrices with $k$ rows and $n$ columns and exactly one 1-cell in each column. We assume that the rows of a matrix are numbered bottom-to-top, and the columns are numbered left-to-right. For a word $\sigma$ of length $n$ over the alphabet $[k]$, let $M(\sigma, k)$ be the $k \times n$ matrix with a 1-cell in row $i$ and column $j$ if and only the $j$-th letter of $\sigma$ is equal to $i$.

With this representation, we may use known bijections on fillings of diagrams to obtain directly new equivalences among words. A Ferrers diagram is an array of cells whose columns have nonincreasing length, and the bottom cells of the columns appear in the same row. A filling of a Ferrers diagram is an assignment of zeros and ones into its cells such that every column has exactly one 1-cell. We say that a filling of a Ferrers shape $F$ contains a matrix $M$ if $F$ has a (not necessarily contiguous) rectangular subshape which induces a filling identical to $M$. We will say that two matrices $M$ and $M^{\prime}$ are Ferrersequivalent if for every Ferrers shape $F$ the number of $M$-avoiding fillings is equal to the number of $M^{\prime}$-avoiding fillings. We say that $M$ and $M^{\prime}$ are strongly Ferrers-equivalent if for every Ferrers shape $F$ there is a bijection between $M$-avoiding and $M^{\prime}$-avoiding fillings of $F$ that preserves the number of 1-cells in each row.

The following lemma allows us to translate results about fillings of Ferrers shapes into results about words. The lemma is based on an idea which is often applied in the context of pattern-avoiding permutations [3], graphs [8] or set partitions [9].

For a word $\rho \in[\ell]^{n}$ and an integer $k$, we let $\rho+k$ denote the word obtained by increasing each letter of $\rho$ by $k$.

Lemma 2.1. Let $\tau$ and $\tau^{\prime}$ be two patterns with $k$ letters, let $\rho$ be a pattern with $\ell$ letters. If $M(\tau, k)$ and $M\left(\tau^{\prime}, k\right)$ are strongly Ferrers-equivalent then the two $(k+\ell)$-letter patterns $\tau(\rho+k)$ and $\tau^{\prime}(\rho+k)$ are strongly equivalent. (Here $\tau(\rho+k)$ denotes the concatenation of $\tau$ and $\rho+k$.) 
Proof. Let us write $\sigma=\tau(\rho+k)$ and $\sigma^{\prime}=\tau^{\prime}(\rho+k)$. For a given $m$ and $n$, choose a word $x \in[m]^{n}(\sigma)$, and let $M=M(x, m)$ be its corresponding matrix. Note that $M$ avoids the matrix $M(\sigma, k+\ell)$.

Color the cells of $M$ red and green, where a cell $c$ is green if and only if the submatrix of $M$ strictly to the right and strictly to the top of $c$ contains $M(\rho, \ell)$, otherwise the cell is red. Note that the green cells form a Ferrers diagram and that the nonzero columns of this diagram induce an $M(\tau, k)$-avoiding filling. Using the strong Ferrers-equivalence of $M(\tau, k)$ and $M\left(\tau^{\prime}, k\right)$, we may transform this filling into a $M\left(\tau^{\prime}, k\right)$-avoiding filing. This operation transforms $M$ into a matrix $M^{\prime}$ representing a $\sigma^{\prime}$-avoiding word $x^{\prime}$ with the same content as $x$.

To see that this operation can be inverted, observe that the operation has only modified the filling of the green cells of $M$. Observe also that for every green cell $c$ of $M$, there is a copy of $M(\rho, \ell)$ strictly to the right and strictly above $c$ which only consists of red cells. Thus the red cells of $M$ coincide with the red cells of $M^{\prime}$.

We thus have a bijection showing that $\sigma \stackrel{s}{\sim} \sigma^{\prime}$.

Using known results about Ferrers equivalence [8, 9, 11], we obtain the following equivalences, valid for any pattern $\rho$.

Fact 2.2. $M(12 \cdots k, k)$ is strongly Ferrers equivalent to $M(k(k-1) \cdots 1, k)$ [11]. This implies that $12 \cdots k(\rho+k) \stackrel{s}{\sim} k(k-1) \cdots 1(\rho+k)$.

Fact 2.3. $M\left(2^{i} 12^{j}, 2\right)$ is strongly Ferrers-equivalent to $M\left(12^{i+j}, 2\right)$, for any $i, j \geq 0[9$, Lemma 39]. This implies that $2^{i} 12^{j}(\rho+2) \stackrel{s}{\sim} 12^{i+j}(\rho+2)$.

The above-mentioned results do not account for all the equivalences among wordpatterns of small length. To complete our classification, we need another lemma, whose proof uses an idea that has been previously applied in the context of pattern-avoiding set partitions [9, Theorem 48].

Lemma 2.4. For any $k$, all the patterns that consist of a single symbol '1', a single symbol '3' and $k-2$ symbols '2' are strongly equivalent.

Proof. Let $k$ be fixed. Let $\tau(i, j)$ denote the word of length $k$ whose $i$-th symbol is ' 1 ', the $j$-th symbol is ' 3 ' and the remaining symbols are equal to '2'. Our aim is to show that all the patterns in the set $\{\tau(i, j), i \neq j, 1 \leq i, j \leq k\}$ are strongly equivalent. Since each word is strongly equivalent to its reversal, we only need to deal with the words $\tau(i, j)$ with $i<j$. From Fact 2.3, we deduce that the words $\{\tau(1, j), j=2, \ldots, k\}$ are all strongly equivalent, and the words $\{\tau(i, k), i=1, \ldots, k-1\}$ are all strongly equivalent as well.

To prove the lemma, it suffices to show that for every $i<j<k$, the word $\tau(i, j)$ is strongly equivalent to the word $\tau(i+1, j+1)$. Let $m$ be an integer. We will say that a word $\sigma$ contains $\tau(i, j)$ at level $m$ if there is a pair of symbols $\ell, h$ such that $\ell<m<h$, and such that the word $\sigma$ contains a subword over the alphabet $\{\ell, m, h\}$ which is orderisomorphic to $\tau(i, j)$. For example, the word 132342 contains the pattern 1223 at level 3 (due to the subword 1334), while it avoids 1223 at level 2. 
Assume now that we are given a fixed pair of indices $i, j$, with $i<j<k$, and we want to provide a content-preserving bijection between $\tau(i, j)$-avoiding and $\tau(i+1, j+1)$ avoiding words of length $n$. We will say that a word $\sigma$ is an $m$-hybrid if for every $\bar{m}<m$, the word $\sigma$ avoids $\tau(i, j)$ at level $\bar{m}$, while for every $\widetilde{m} \geq m, \sigma$ avoids $\tau(i+1, j+1)$ at level $\widetilde{m}$. We will present, for any $m \geq 1$, a content-preserving bijection between $m$-hybrids and $(m+1)$-hybrids. By composing these bijections, we obtain the required bijection between $\tau(i, j)$-avoiding and $\tau(i+1, j+1)$-avoiding words.

Let $m \geq 1$ be fixed. Let $\sigma$ be an arbitrary word. A letter of $\sigma$ is called low if it is smaller than $m$, and a letter is called high if it is greater than $m$. A low cluster of $\sigma$ is a maximal block of consecutive low symbols of $\sigma$. A high cluster is defined analogously. Thus, every symbol of $\sigma$ different from $m$ belongs to a unique cluster. The landscape of $\sigma$ is a word over the alphabet $\{\mathrm{L}, m, \mathrm{H}\}$ obtained by replacing every low cluster of $\sigma$ by a single symbol L, and every high cluster of $\sigma$ by a single symbol H. Note that $\sigma$ contains $\tau(i, j)$ at level $m$ if and only if the landscape of $\sigma$ contains the subsequence $m^{i-1} \mathrm{~L}^{j-i-1} \mathrm{H} m^{k-j}$.

We will now describe the bijection between $m$-hybrids and $(m+1)$-hybrids. Let $\sigma$ be an $m$-hybrid word, let $X$ be its landscape. We split $X$ into three parts $X=P m S$, where $P$ is the prefix of $X$ formed by all the symbols of $X$ that appear before the first occurrence of $m$ in $X$, and $S$ is the suffix of all the symbols that appear after the first occurrence of $m$. Let us define a word $X^{\prime}$ by $X^{\prime}=S m P$. Note that $X^{\prime}$ contains a subsequence $m^{i-1} \mathrm{Lm}^{j-i-1} \mathrm{H} m^{k-j}$ if and only if $X$ contains a subsequence $m^{i} \mathrm{Lm}^{j-i-1} \mathrm{H} m^{k-j-1}$. Thus, since $X$ is a landscape of a word that avoids $\tau(i+1, j+1)$ at level $m$, we know that any word with landscape $X^{\prime}$ must avoid $\tau(i, j)$ at level $m$.

Let us define a word $\sigma^{\prime}$ by the following three rules.

1. The word $\sigma^{\prime}$ has landscape $X^{\prime}$.

2. For any $p$, the $p$-th low cluster of $\sigma^{\prime}$ consists of the same sequence of symbols as the $p$-th low cluster of $\sigma$.

3. For any $q$, the $q$-th high cluster of $\sigma^{\prime}$ consists of the same sequence of symbols as the $q$-th high cluster of $\sigma$.

Clearly, there is a unique word $\sigma^{\prime}$ satisfying these properties. Note that the subsequence of all the low symbols of $\sigma$ is the same as the subsequence of all the low symbols of $\sigma^{\prime}$, and these sequences are partitioned into low clusters in the same way. An analogous property holds for the high symbols too.

We claim that $\sigma^{\prime}$ is an $(m+1)$-hybrid. We have already pointed out that $\sigma^{\prime}$ avoids $\tau(i, j)$ at level $m$. Let us now argue that $\sigma^{\prime}$ avoids $\tau(i, j)$ at level $\bar{m}$, for every $\bar{m}<m$. For contradiction, assume that $\sigma^{\prime}$ contains a subsequence $T=\bar{m}^{i-1} \ell \bar{m}^{j-i-1} h \bar{m}^{k-j}$, for some $\ell<\bar{m}<h$. If $h<m$, then all the symbols of $T$ are low, and since $\sigma$ has the same subsequence of low symbols as $\sigma^{\prime}$, we know that $\sigma$ also contains $T$ as a subsequence, contradicting the assumption that $\sigma$ is an $m$-hybrid.

Assume now that $h \geq m$. Let $x$ and $y$ be the two symbols adjacent to $h$ in the sequence $T$ (note that $h$ is not the last symbol of $T$, so $x$ and $y$ are well defined). Both $x$ and $y$ are 
low, and they belong to distinct low clusters of $\sigma^{\prime}$, because the symbol $h$ is not low. Since the low symbols of $\sigma$ are the same as the low symbols of $\sigma^{\prime}$, and they are partitioned into clusters in the same way, we know that $\sigma$ contains a subsequence $\bar{m}^{i-1} \ell \bar{m}^{j-i-1} h^{\prime} \bar{m}^{k-j}$, where $h^{\prime}$ is a non-low symbol. This shows that $\sigma$ contains $\tau(i, j)$ at level $\bar{m}$, which is impossible, because $\sigma$ is an $m$-hybrid.

By an analogous argument, we may show that $\sigma^{\prime}$ avoids $\tau(i+1, j+1)$ at any level $\widetilde{m}>m$. We conclude that the mapping described above transforms an $m$-hybrid $\sigma$ into an $(m+1)$-hybrid $\sigma^{\prime}$. It is clear that the mapping is reversible and provides the required bijection between $m$-hybrids and $(m+1)$-hybrids.

\section{Appendix A: the w-equivalence classes}

In Tables 1, 2 and 3, we list the nontrivial w-equivalence classes of patterns of size 4, 5 and 6, respectively. From each symmetry class (i.e., a class generated by reversal and complement of a single pattern) we only list the lexicographically minimal pattern. We only list the w-equivalence classes that have at least two nonsymmetric elements.

\begin{tabular}{|l|l|l|l|}
\hline $1123 \stackrel{w}{\sim} 1132$ & $1112 \stackrel{w}{\sim} 1121$ & $1234 \stackrel{w}{\sim} 1243 \stackrel{w}{\sim} 1432 \stackrel{w}{\sim} 2143$ & $1223 \stackrel{w}{\sim} 1232 \stackrel{w}{\sim} 1322 \stackrel{w}{\sim} 2132$ \\
\hline
\end{tabular}

Table 1: w-equivalence classes of patterns of size 4

\begin{tabular}{|l|l|l|l|}
\hline $12435 \stackrel{w}{\sim} 13254$ & $12443 \stackrel{w}{\sim} 21143$ & $11234 \stackrel{w}{\sim} 11243 \stackrel{w}{\sim} 11432$ & $12223 \stackrel{w}{\sim} 12232 \stackrel{w}{\sim} 12322 \stackrel{w}{\sim} 13222 \stackrel{w}{\sim} 21232 \sim 21322$ \\
\hline $12134 \stackrel{w}{\sim} 12143$ & $11123 \stackrel{w}{\sim} 11132$ & $11223 \stackrel{w}{\sim} 11232 \stackrel{w}{\sim} 11322$ & $12345 \stackrel{w}{\sim} 12354 \stackrel{w}{\sim} 12543 \sim 15432 \sim 21354 \sim 21543$ \\
\hline $12534 \stackrel{w}{\sim} 21534$ & $12453 \stackrel{w}{\sim} 21453$ & $11112 \stackrel{w}{\sim} 11121 \stackrel{w}{\sim} 11211$ & $12234 \stackrel{w}{\sim} 12243 \stackrel{w}{\sim} 12343 \stackrel{w}{\sim} 12433 \stackrel{w}{\sim} 21243 \stackrel{w}{\sim} 21433$ \\
\hline
\end{tabular}

Table 2: w-equivalence classes of patterns of size 5

\begin{tabular}{|c|c|c|c|c|}
\hline $124433 \stackrel{w}{\sim} 214433$ & $112234 \stackrel{w}{\sim} 112243$ & $125354 \stackrel{w}{\sim} 213154$ & $111223 \stackrel{w}{\sim} 111232 \stackrel{w}{\sim} 111322$ & $112223 \stackrel{w}{\sim} 112232 \sim{ }^{w} \sim 12322 \sim{ }^{w} \sim 13222$ \\
\hline $124353 \stackrel{w}{\sim} 214353$ & $124535 \stackrel{w}{\sim} 131254$ & $125334 \stackrel{w}{\sim} 215334$ & $112334 \stackrel{w}{\sim} 112343 \stackrel{w}{\sim} 112433$ & $112345 \stackrel{w}{\sim} 112354 \stackrel{w}{\sim} 112543 \stackrel{w}{\sim} 115432$ \\
\hline $124453 \stackrel{w}{\sim} 214453$ & $124553 \stackrel{w}{\sim} 214553$ & $113245 \stackrel{w}{\sim} 113254$ & $123554 \stackrel{w}{\sim} 211354 \stackrel{w}{\sim} 211543$ & \multirow{2}{*}{$\begin{array}{l}122334 \stackrel{w}{\sim} 122343 \stackrel{w}{\sim} 122433 \stackrel{w}{\sim} 212343 \\
\stackrel{w}{\sim} 212433 \stackrel{w}{\sim} 221433\end{array}$} \\
\hline $124653 \sim 214653$ & $126354 \stackrel{w}{\sim} 216354$ & $125463 \stackrel{w}{\sim} 215463$ & $122435 \stackrel{w}{\sim} 132454 \stackrel{w}{\sim} 132544$ & \\
\hline $126435 \sim 216435$ & $125436 \stackrel{w}{\sim} 143265$ & $122134 \stackrel{w}{\sim} 122143$ & $121345 \stackrel{w}{\sim} 121354 \stackrel{w}{\sim} 121543$ & \multirow{2}{*}{ 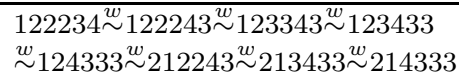 } \\
\hline $125634 \sim 215634$ & $124563 \stackrel{w}{\sim} 214563$ & $126453 \stackrel{w}{\sim} 216453$ & $111112 \stackrel{w}{\sim} 111121 \sim 111211$ & \\
\hline $126534 \sim 216534$ & $126345 \stackrel{w}{\sim} 216345$ & $124343 \stackrel{w}{\sim} 212143$ & $123645 \stackrel{w}{\sim} 213645 \stackrel{w}{\sim} 231654$ & \multirow{3}{*}{$\begin{array}{l}122223 \stackrel{w}{\sim} 122232 \stackrel{w}{\sim} 122322 \stackrel{w}{\sim} 123222 \\
w_{1} 132222 \stackrel{w}{\sim} 212232 \stackrel{w}{\sim} 212322 \sim 213222 \\
{ }_{w}^{\sim} 221322\end{array}$} \\
\hline $123443 \stackrel{w}{\sim} 211243$ & $125346 \stackrel{w}{\sim} 134265$ & $121234 \sim 121243$ & $123564 \stackrel{w}{\sim} 213564 \stackrel{w}{\sim} 312654$ & \\
\hline $125643 \stackrel{w}{\sim} 215643$ & $125543 \stackrel{w}{\sim} 215543$ & $123543 \stackrel{w}{\sim} 213543$ & $65 \sim 132654$ & \\
\hline $125435 \sim 132154$ & $124354 \stackrel{w}{\sim} 213254$ & $123145 \sim 123154$ & $124356 \stackrel{w}{\sim} 124365 \stackrel{w}{\sim} 214365$ & \multirow{3}{*}{$\begin{array}{l}122345 \stackrel{w}{\sim} 122354 \stackrel{w}{\sim} 122543 \stackrel{w}{\sim} 123454 \\
\stackrel{w}{\sim} 123544 \stackrel{w}{\sim} 212354 \stackrel{w}{\sim} 212543 \stackrel{w}{\sim} 213544 \\
\stackrel{w}{\sim}_{221543}\end{array}$} \\
\hline $125344 \sim 215344$ & $124543 \stackrel{w}{\sim} 214543$ & $124443 \stackrel{w}{\sim} 211143$ & $343 \stackrel{w}{\sim} 121433$ & \\
\hline $121134 \sim 121143$ & $112134 \stackrel{w}{\sim} 112143$ & $124533 \stackrel{w}{\sim} 214533$ & $122534 \stackrel{w}{\sim} 212534 \stackrel{w}{\sim} 221534$ & \\
\hline $123435 \stackrel{w}{\sim} 132354$ & $123245 \stackrel{w}{\sim} 123254$ & $125534 \stackrel{w}{\sim} 215534$ & $111234 \stackrel{w}{\sim} 111243 \stackrel{w}{\sim} 111432$ & \multirow{3}{*}{$\begin{array}{l}123456 \stackrel{w}{\sim} 123465 \stackrel{w}{\sim} 123654 \stackrel{w}{\sim} 126543 \\
\stackrel{w}{\sim} 165432 \sim 213465 \stackrel{w}{\sim} 213654 \stackrel{w}{\sim} 216543 \\
\stackrel{w}{\sim} 321654\end{array}$} \\
\hline $124335 \stackrel{w}{\sim} 133254$ & $124435 \stackrel{w}{\sim} 132254$ & $124635 \stackrel{w}{\sim} 214635$ & $123345 \stackrel{w}{\sim} 123354 \stackrel{w}{\sim} 213354$ & \\
\hline $125453 \stackrel{w}{\sim} 215453$ & $125434 \stackrel{w}{\sim} 215434$ & $111123 \sim{ }^{w} \sim 11132$ & $122453 \stackrel{w}{\sim} 212453 \stackrel{w}{\sim} 221453$ & \\
\hline $123534 \sim 213534$ & $125433 \stackrel{w}{\sim} 215433$ & $125364 \stackrel{w}{\sim} 215364$ & $122443 \stackrel{w}{\sim} 211343 \stackrel{w}{\sim} 211433$ & \\
\hline
\end{tabular}

Table 3: w-equivalence classes of patterns of size 6 


\section{Appendix B: p-equivalence and c-equivalence classes}

In Tables 4 and 5, we list the nontrivial c-equivalence classes for patterns of size 4 and 5, respectively. For patterns of these sizes, the c-equivalence classes coincide with pequivalence classes. A symmetry class of a pattern is generated by the reversal operation. We again consider only one representative of each symmetry class, and we only list the c-equivalence classes with at least two nonsymmetric elements.

\begin{tabular}{|c|c|c|}
\hline $1234 \stackrel{c}{\sim} 1243 \stackrel{c}{\sim} 1432 \stackrel{c}{\sim} 2134 \stackrel{c}{\sim} 2143 \stackrel{c}{\sim} 3214$ & $1112 \stackrel{\mathcal{C}}{\sim} 1121$ & $1123 \stackrel{c}{\sim} 1132$ \\
\hline $1223 \stackrel{c}{\sim} 1232 \stackrel{c}{\sim} 1322 \stackrel{c}{\sim}^{\sim} 2123 \stackrel{c}{\sim}^{\sim} 2132 \stackrel{c}{\sim}^{\sim} 2213$ & $123 \stackrel{C}{\sim}_{2}^{\sim} 2133$ & $1222 \stackrel{c}{\sim} 2122$ \\
\hline
\end{tabular}

Table 4: c-equivalence classes of patterns of size 4

\begin{tabular}{|c|c|c|c|}
\hline $11123 \stackrel{c}{\sim} 11132$ & $12333 \stackrel{C}{\sim} 21333$ & $11112 \stackrel{c}{\sim} 11121 \stackrel{c}{\sim} 11211$ & 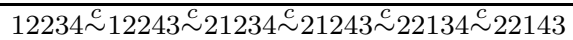 \\
\hline $12134 \stackrel{c}{\sim} 12143$ & $12443 \stackrel{c}{\sim} 21$ & $11223 \stackrel{c}{\sim} 1$ & $34 \stackrel{c}{\sim} 21343 \stackrel{c}{\sim} 21433$ \\
\hline $12434 \stackrel{C}{\sim} 21434$ & $4 \stackrel{C}{\sim} 21$ & $12222 \stackrel{C}{\sim} 2$ & \multirow{2}{*}{$\begin{array}{l}12223 \stackrel{c}{\sim} 12232 \stackrel{c}{\sim} 12322 \stackrel{c}{\sim} 13222 \stackrel{c}{\sim} 21223 \stackrel{c}{\sim} 21232 \\
\stackrel{c}{\sim} 21322 \stackrel{c}{\sim} 22123 \stackrel{c}{\sim} 22132 \stackrel{c}{\sim} 22213\end{array}$} \\
\hline $12435 \stackrel{C}{\sim} 21435$ & $12453 \stackrel{\mathcal{C}}{\sim} 21453$ & $11234 \stackrel{\stackrel{C}{\sim} 11}{1}$ & \\
\hline $13245 \stackrel{c}{\sim} 13254$ & $23145 \stackrel{\mathcal{C}}{\sim} 23154$ & $12233 \stackrel{\mathcal{C}}{\sim} 21$ & \multirow{2}{*}{$\begin{array}{l}12345 \stackrel{\mathcal{c}}{\sim} 12354 \stackrel{\mathcal{c}}{\sim} 12543 \stackrel{\mathcal{c}}{\sim} 15432 \stackrel{\mathcal{c}}{\sim} 21345 \stackrel{c}{\sim} 21354 \\
\stackrel{c}{\sim} 21543 \stackrel{c}{\sim} 32145 \stackrel{c}{\sim} 32154 \stackrel{c}{\sim} 43215\end{array}$} \\
\hline $21134 \stackrel{C}{\sim} 21143$ & $31245 \stackrel{C}{\sim} 31254$ & $12344 \stackrel{C}{\sim} 21344 \stackrel{C}{\sim} 32144$ & \\
\hline
\end{tabular}

Table 5: c-equivalence classes of patterns of size 5

\section{References}

[1] M. Albert, R. Aldred, M.D. Atkinson, C. Handley, And D. Holton, Permutations of a multiset avoiding permutations of length 3, Europ. J. Combin. 22 (2001) 1021-1031.

[2] N. Alon And E. Friedgut, On the number of permutations avoiding a given pattern, J. Combin. Theory Series A 89 (2000) 133-140.

[3] J. Backelin, J. West, And G. Xin, Wilf-equivalence for singleton classes, Adv. Appl. Math. 32:2 (2007) 133-148.

[4] A. Burstein, Enumeration of words with forbidden patterns, Ph.D. thesis, University of Pennsylvania, 1998.

[5] A. Burstein and T. Mansour, Words restricted by patterns with at most 2 distinct letters, Electron. J. Combin. 9:2 (2002), \#R3.

[6] S.-P. EU, T.-S. FU, AND C.-J. LAI, On the enumeration of parking functions by leading terms, Adv. in Appl. Math. 35:4 (2005) 392-406.

[7] S. Heubach, T. Mansour, and A. Munagi, Avoiding permutation patterns of type $(2,1)$ in compositions, preprint.

[8] A. DE MIER, $k$-noncrossing and $k$-nonnesting graphs and fillings of Ferrers diagrams, Electronic Notes in Discrete Mathematics 28 (2007) 3-10. 
[9] V. Jelínek and T. Mansour, On pattern-avoiding partitions Elect. J. Combin. 15:1 (2008), \#R39.

[10] M. Klazar, The Füredi-Hajnal conjecture implies the Stanley-Wilf conjecture, Formal power series and algebraic combinatorics (Moscow,2000), Springer, Berlin (2000) 250-255.

[11] C. Krattenthaler, Growth diagrams, and increasing and decreasing chains in fillings of Ferrers shapes, Adv. Appl. Math. 37:3 (2006) 404-431.

[12] A. Rattan, Permutation factorizations and prime parking functions, Ann. Comb. 10:2 (2006) 237-254.

[13] A. Regev, Asymptotics of the number of $k$-words with an $\ell$-descent, Elect. J. Combin. 5 (1998), \#R15.

[14] C. D. Savage And H. S. Wilf, Pattern avoidance in compositions and multiset permutations, Adv. Appl. Math. 36:2 (2006) 194-201.

[15] C. ZARA, Parking functions, stack-sortable permutations, and spaces of paths in the Johnson graph, Electron. J. Combin. 9:2 (2002/03) \#R11.

[16] http://math.haifa.ac.il/toufik/enumerative/tablewords456.html.

[17] http://math.haifa.ac.il/toufik/enumerative/tablecomposition45.html.

[18] http://math.haifa.ac.il/toufik/enumerative/tableparking45.html.

[19] http://math.haifa.ac.il/toufik/program/classification_words.html

[20] http://math.haifa.ac.il/toufik/program/classification_compositions.html

[21] http://math.haifa.ac.il/toufik/program/classification_parkings.html 